\title{
Correction to: Examining the Interdependence of Parent-adolescent Acculturation Gaps on Acculturation-based Conflict: Using the Actor-Partner Interdependence Model
}

Meme Wang-Schweig $\mathbb{D}^{1} \cdot$ Brenda A. Miller $^{1}$

Published online: 29 December 2018

(c) Springer Science+Business Media, LLC, part of Springer Nature 2019

Correction to: Journal of Youth and Adolescence (2018) https://doi.org/10.1007/s10964-018-0948-9

The original version of this article unfortunately contained an alignment error in the tables. The corrected Tables 1 and 2 are presented with this erratum.

The original article has been corrected.

The original article can be found online at https://doi.org/10.1007/ s10964-018-0948-9.

Brenda A. Miller

bmiller@prev.org

1 Prevention Research Center/Pacific Institute for Research and Evaluation, 2150 Shattuck Avenue, Suite 601, Berkeley, CA 94704-1365, USA 
Table 1 Summary of correlations, means, and standard deviations for scores on adolescent and parent acculturation, cultural maintenance, and perceived acculturation-based conflict variables

\begin{tabular}{lllllllll}
\hline Measure & 1 & 2 & 3 & 4 & 5 & 6 & $M$ & $S D$ \\
\hline 1. Adolescent acculturation & - & .02 & $.26^{* *}$ & -.02 & 0.04 & $-.24^{* *}$ & 3.39 & 0.76 \\
2. Adolescent cultural maintenance & & - & .08 & .07 & -.11 & $-.29^{* *}$ & 3.45 & 0.68 \\
3. Parent acculturation & & & - & $-.18^{*}$ & $-.30^{* *}$ & $-.17^{*}$ & 2.74 & 0.63 \\
4. Parent cultural maintenance & & & & - & .07 & .12 & 3.94 & 0.50 \\
5. Adolescent perceived conflict & & & & - & $.16^{* *}$ & 2.55 & 1.47 \\
6. Parent perceived conflict & & & & & - & 1.88 & 0.65 \\
\hline
\end{tabular}

$* p<.05$, two-tailed; $* * p<.01$, two-tailed

Table 2 Actor-partner interaction effects on perceived acculturationbased conflict ( $n=187$ dyads)

\begin{tabular}{lccr}
\hline APIM parameters & $\beta$ & S.E. & \multicolumn{1}{l}{$t$} \\
\hline Acculturation & & & \\
Actor $\times$ partner effects & $-0.18^{*}$ & 0.09 & -2.06 \\
$\quad$ Adolescent & -0.23 & 0.23 & -0.99 \\
$\quad$ Parent & -0.03 & 0.10 & -0.25 \\
|Actor-partnerl effects & $0.21^{*}$ & 0.08 & 2.52 \\
Adolescent & $0.54^{*}$ & 0.25 & 2.19 \\
Parent & 0.00 & 0.11 & -0.14 \\
Cultural maintenance & & & \\
Actor $\times$ partner effects & $-0.51^{* * *}$ & 0.14 & -3.56 \\
Adolescent & $-0.97^{*}$ & 0.38 & -2.58 \\
Parent & $-0.33^{*}$ & 0.16 & -2.06 \\
IActor-partnerl effects & $0.25^{*}$ & 0.10 & 2.58 \\
Adolescent & 0.15 & 0.28 & 0.51 \\
Parent & 0.12 & 0.12 & 0.98 \\
\hline
\end{tabular}

Models adjusted for demographic variables, including age and gender of adolescent, age of parent, educational level of parent, household income, and time lived in the US since year of immigration

$* p<.05 ; * * * p<.001$ 